\title{
Solitons and Other Exact Solutions for Two Nonlinear PDEs in Mathematical Physics Using the Generalized Projective Riccati Equations Method
}

\author{
A. M. Shahoot, ${ }^{1}$ K. A. E. Alurrfi $(1),{ }^{2}$ I. M. Hassan, ${ }^{3}$ and A. M. Almsri ${ }^{4}$ \\ ${ }^{1}$ Department of Physics, Faculty of Science, Elmergib University, Khoms, Libya \\ ${ }^{2}$ Department of Mathematics, Faculty of Science, Elmergib University, Khoms, Libya \\ ${ }^{3}$ Department of Mathematics, Faculty of Science, Tripoli University, Tripoli, Libya \\ ${ }^{4}$ Department of Mathematical Sciences, The Libyan Academy, Tripoli, Libya
}

Correspondence should be addressed to K. A. E. Alurrfi; alurrfi@yahoo.com

Received 26 December 2017; Accepted 12 March 2018; Published 22 April 2018

Academic Editor: Zhijun Qiao

Copyright (C) 2018 A. M. Shahoot et al. This is an open access article distributed under the Creative Commons Attribution License, which permits unrestricted use, distribution, and reproduction in any medium, provided the original work is properly cited.

\begin{abstract}
We apply the generalized projective Riccati equations method with the aid of Maple software to construct many new soliton and periodic solutions with parameters for two higher-order nonlinear partial differential equations (PDEs), namely, the nonlinear Schrödinger (NLS) equation with fourth-order dispersion and dual power law nonlinearity and the nonlinear quantum ZakharovKuznetsov (QZK) equation. The obtained exact solutions include kink and antikink solitons, bell (bright) and antibell (dark) solitary wave solutions, and periodic solutions. The given nonlinear PDEs have been derived and can be reduced to nonlinear ordinary differential equations (ODEs) using a simple transformation. A comparison of our new results with the well-known results is made. Also, we drew some graphs of the exact solutions using Maple. The given method in this article is straightforward and concise, and it can also be applied to other nonlinear PDEs in mathematical physics.
\end{abstract}

\section{Introduction}

The investigation of exact traveling wave solutions to nonlinear PDEs plays an important role in the study of nonlinear physical phenomena. Nonlinear waves appear in various scientific fields, especially in physics such as fluid mechanics, plasma physics, optical fibers, and solid state physics. In recent years, many powerful tools have been established to determine soliton and periodic wave solutions of nonlinear PDEs, such as the $\left(G^{\prime} / G\right)$-expansion method [1-6], the extended auxiliary equation method $[7,8]$, the new mapping method [9-11], the generalized projective Riccati equations method [12-17], and the $\left(G^{\prime} / G, 1 / G\right)$-expansion method [18]. Conte and Musette [12] presented an indirect method to find solitary wave solutions of some nonlinear PDEs that can be expressed as polynomials in two elementary functions which satisfy a projective Riccati equation [19]. This method has been applied to many nonlinear PDEs and the solitary wave solutions of these equations can be found in [13-17].
Recently, Yan [16] has been given a generalization of Conte and Musette's method.

The objective of this article is to use the generalized projective Riccati equations method [13-17] to construct the soliton and periodic solutions of the following two higherorder nonlinear PDEs.

(1) The nonlinear Schrödinger (NLS) equation with fourth-order dispersion and dual power law nonlinearity [5] is

$$
i q_{t}+a q_{x x}-b q_{x x x x}+c\left(|q|^{2 m}+k_{1}|q|^{4 m}\right) q=0
$$

which describes the propagation of optical pulse in a medium, and $q(x, t)$ is the slowly varying envelope of the electromagnetic field, where $a, b, c$ are real numbers. If $b=0$, (1) reduces to the NLS. In addition if $m=1$, (1) reduces to parabolic law nonlinearity, which has been discussed in [20] using two direct algebraic methods. The coefficient of $a$ represents the group velocity dispersion (GVD), while the 
coefficient of $c$ represents the self-phase modulation (SPM) with dual power law nonlinearity. The constant $k_{1}$ binds the two nonlinear terms and the exponent $m$ governs the power law. Also, the coefficients of $b$ are the fourth-order dispersion terms. Equation (1) has been studied in [5] using five different techniques, namely, the $\left(G^{\prime} / G\right)$-expansion method, the improved Sub-ODE method, the extended auxiliary equation method, the new mapping method, and the Jacobi elliptic function method.

(2) The nonlinear quantum Zakharov-Kuznetsov (QZK) equation $[6,21-23]$ is

$$
\frac{\partial U}{\partial T}+A U \frac{\partial U}{\partial Z}+B \frac{\partial^{3} U}{\partial Z^{3}}+C \frac{\partial}{\partial Z}\left(\frac{\partial^{2}}{\partial X^{2}}+\frac{\partial^{2}}{\partial Y^{2}}\right) U=0,
$$

which arises in quantum magnetoplasma, where $A, B, C$ are constants. Equation (2) has been derived in [21] using the reductive perturbation technique and in [22] using a series of transformations method. Here $U$ is the electrostatic potential, which $X, Y, Z, T$ are the stretched space-time coordinates which are defined in [21]. Moslem et al. [21] have derived (2) for electron-ion quantum plasma and solitary explosive and periodic solutions are presented. In [23], the authors applied the auxiliary equation method and Hirota bilinear method to study (2) and some types of exact solutions are obtained. Recently Zayed and Alurrfi have discussed (2) in [6] using the extended generalized $\left(G^{\prime} / G\right)$-expansion method with the Jacobi elliptic equation and determined its exact traveling wave solutions.

This paper is organized as follows: in Section 2, the description of the well-known generalized projective Riccati equations method is given. In Section 3, we use the given method described in Section 2, to find new soliton and periodic solutions of the NLS equation (1) and the QZK equation (2). In Section 4, we draw some figures for some solutions of (1). In Section 5, some conclusions are obtained.

\section{Description of the Generalized Projective Riccati Equations Method}

Consider a nonlinear PDE in the following form:

$$
P\left(u, u_{x}, u_{t}, u_{x x}, u_{t t}, \ldots\right)=0,
$$

where $u=u(x, t)$ is an unknown function, $P$ is a polynomial in $u(x, t)$, and its partial derivatives in which the highestorder derivatives and nonlinear terms are involved. Let us now give the main steps of the generalized projective Riccati equations method [13-17].

Step 1. We use the following transformation:

$$
u(x, t)=u(\xi), \quad \xi=x-v t,
$$

to reduce (3) to the following nonlinear ODE:

$$
H\left(u, u^{\prime}, u^{\prime \prime}, \ldots\right)=0,
$$

where $v$ is velocity of the propagation, $H$ is a polynomial of $u(\xi)$ and its total derivatives $u^{\prime}(\xi), u^{\prime \prime}(\xi), \ldots$ and ${ }^{\prime}=d / d \xi$.
Step 2. We suppose that the solution of (5) has the following form:

$$
u(\xi)=A_{0}+\sum_{i=1}^{N} \sigma^{i-1}(\xi)\left[A_{i} \sigma(\xi)+B_{i} \tau(\xi)\right]
$$

where $A_{0}, A_{i}$, and $B_{i}$ are constants to be determined. The functions $\sigma(\xi)$ and $\tau(\xi)$ satisfy the ODEs:

$$
\begin{aligned}
\sigma^{\prime}(\xi) & =\varepsilon \sigma(\xi) \tau(\xi), \\
\tau^{\prime}(\xi) & =R+\varepsilon \tau^{2}(\xi)-\mu \sigma(\xi), \quad \varepsilon= \pm 1,
\end{aligned}
$$

where

$$
\tau^{2}(\xi)=-\varepsilon\left(R-2 \mu \sigma(\xi)+\frac{\mu^{2}+r}{R} \sigma^{2}(\xi)\right),
$$

and here $r= \pm 1$ and $R, \mu$ are nonzero constants.

If $R=\mu=0$, (5) has the formal solution:

$$
u(\xi)=\sum_{i=0}^{N} A_{i} \tau^{i}(\xi)
$$

where $\tau(\xi)$ satisfies the nonlinear ODE:

$$
\tau^{\prime}(\xi)=\tau^{2}(\xi) .
$$

Step 3. The positive integer number $N$ in (6) must be determined by using the homogeneous balance between the highest-order derivatives and the highest nonlinear terms in (5).

Step 4. Substitute (6) along with (7) and (8) into (5). Collecting all terms of the same order of $\sigma^{j}(\xi) \tau^{i}(\xi)(j=0,1, ; i=$ $0,1)$. Setting each coefficient to zero yields a set of algebraic equations which can be solved to find the values of $A_{0}, A_{i}, B_{i}$, $v, \mu$, and $R$.

Step 5. It is well-known [13-17] that (7) admits the following solutions.

Case 1. When $\varepsilon=-1, r=-1, R>0$,

$$
\begin{gathered}
\sigma_{1}(\xi)=\frac{R \operatorname{sech}(\sqrt{R} \xi)}{\mu \operatorname{sech}(\sqrt{R} \xi)+1}, \\
\tau_{1}(\xi)=\frac{\sqrt{R} \tanh (\sqrt{R} \xi)}{\mu \operatorname{sech}(\sqrt{R} \xi)+1} .
\end{gathered}
$$

Case 2. When $\varepsilon=-1, r=1, R>0$,

$$
\begin{gathered}
\sigma_{2}(\xi)=\frac{R \operatorname{csch}(\sqrt{R} \xi)}{\mu \operatorname{csch}(\sqrt{R} \xi)+1}, \\
\tau_{2}(\xi)=\frac{\sqrt{R} \operatorname{coth}(\sqrt{R} \xi)}{\mu \operatorname{csch}(\sqrt{R} \xi)+1} .
\end{gathered}
$$


Case 3. When $\varepsilon=1, r=-1, R>0$,

$$
\begin{gathered}
\sigma_{3}(\xi)=\frac{R \sec (\sqrt{R} \xi)}{\mu \sec (\sqrt{R} \xi)+1}, \\
\tau_{3}(\xi)=\frac{\sqrt{R} \tan (\sqrt{R} \xi)}{\mu \sec (\sqrt{R} \xi)+1}, \\
\sigma_{4}(\xi)=\frac{R \csc (\sqrt{R} \xi)}{\mu \csc (\sqrt{R} \xi)+1}, \\
\tau_{4}(\xi)=-\frac{\sqrt{R} \cot (\sqrt{R} \xi)}{\mu \csc (\sqrt{R} \xi)+1} .
\end{gathered}
$$

Case 4. When $R=\mu=0$,

$$
\begin{aligned}
& \sigma_{5}(\xi)=\frac{C}{\xi}, \\
& \tau_{5}(\xi)=\frac{1}{\varepsilon \xi},
\end{aligned}
$$

where $C$ is a nonzero constant.

Step 6. Substituting the values of $A_{0}, A_{i}, B_{i}, v, \mu$, and $R$ as well as the solutions (11)-(14) into (6), we obtain the exact solutions of (3).

\section{Applications}

In this section, we apply the generalized projective Riccati equations method described in Section 2 to find many new soliton and periodic solutions of (1) and (2) in the following subsections.

3.1. On Solving (1) Using the Method of Section 2. In order to solve (1), we assume that the solution of (1) has the following form:

$$
q(x, t)=\phi(\xi) e^{i \mathrm{Q}(x, t)}, \quad i=\sqrt{-1},
$$

where $\phi(\xi)$ is the amplitude portion which is a real function of $\xi$, while $Q(x, t)$ is the phase portion of the soliton. It is assumed that $\xi$ and $Q(x, t)$ are given by

$$
\begin{gathered}
\xi=x-v t, \\
Q(x, t)=-k x+\omega t+\theta,
\end{gathered}
$$

where $v, k, \omega$, and $\theta$ are constants, such that $v$ is the velocity of the soliton, $\omega$ is the frequency of the soliton, $k$ is the wave number, and $\theta$ is a phase constant.

Substituting (15) into (1) and separating the real and imaginary parts, we get

$$
\begin{aligned}
& \text { Re: }-\left(\omega+a k^{2}+b k^{4}\right) \phi+\left(a+6 b k^{2}\right) \phi^{\prime \prime}-b \phi^{\prime \prime \prime \prime} \\
& \quad+c\left(\phi^{2 m+1}+k_{1} \phi^{4 m+1}\right)=0 \\
& \operatorname{Im}:-\left(v+2 a k+4 b k^{3}\right) \phi^{\prime}+4 b k \phi^{\prime \prime \prime}=0
\end{aligned}
$$

and, differentiating (18) and substituting the resulting equation in (17), we have the following nonlinear ODE:

$$
a_{1} \phi^{\prime \prime}-b_{1} \phi+c_{1}\left(\phi^{2 m+1}+k_{1} \phi^{4 m+1}\right)=0,
$$

where $a_{1}, b_{1}$, and $c_{1}$ are given by

$$
\begin{aligned}
& a_{1}=2 a k+20 b k^{3}-v, \\
& b_{1}=-4 k\left(\omega+a k^{2}+b k^{4}\right), \\
& c_{1}=4 k c .
\end{aligned}
$$

Balancing $\phi^{\prime \prime}$ with $\phi^{4 m+1}$ in (19), then the following relation is attained:

$$
N+2=N(4 m+1) \Longrightarrow N=\frac{1}{2 m}
$$

Since the balance number $N$ is not integer, then we use the following new transformation:

$$
\phi(\xi)=[u(\xi)]^{1 / 2 m}
$$

where $u$ is a new function of $\xi$. Substituting (22) into (19), we have the new equation

$$
\begin{aligned}
& 2 a_{1} m u u^{\prime \prime}+a_{1}(1-2 m)\left(u^{\prime}\right)^{2}+4 b_{1} m^{2} u^{2}+4 c_{1} m^{2} u^{3} \\
& +4 k_{1} c_{1} m^{2} u^{4}=0 .
\end{aligned}
$$

Balancing $u u^{\prime \prime}$ and $u^{4}$ in (23), then the following relation is obtained:

$$
N+N+2=4 N \Longrightarrow N=1
$$

From (6) the formal solution of (23) has the following form:

$$
u(\xi)=A_{0}+A_{1} \sigma(\xi)+B_{1} \tau(\xi)
$$

where $A_{0}, A_{1}$, and $B_{1}$ are constants to be determined such that $A_{1} \neq 0$ or $B_{1} \neq 0$.

Substituting (25) into (23) and using (7) and (8), the lefthand side of (23) becomes a polynomial in $\sigma(\xi)$ and $\tau(\xi)$. Setting the coefficients of this polynomial to be zero yields the following system of algebraic equations:

$$
\begin{aligned}
\sigma^{4} & : 4 m^{2} A_{1}^{4} c_{1} k_{1}-\frac{1}{R} \varepsilon\left(\mu^{2}+r\right)\left(24 c_{1} k_{1} m^{2} A_{1}^{2} B_{1}^{2}\right. \\
& \left.+4 a_{1} m \varepsilon^{2} A_{1}^{2}+(1-2 m) a_{1} \varepsilon^{2} A_{1}^{2}\right)+\frac{1}{R^{2}} \varepsilon^{2}\left(\mu^{2}\right. \\
& +r)^{2}\left(4 c_{1} k_{1} m^{2} B_{1}^{4}+4 a_{1} m \varepsilon^{2} B_{1}^{2}+(1-2 m) a_{1} \varepsilon^{2} B_{1}^{2}\right) \\
& =0
\end{aligned}
$$




$$
\begin{aligned}
& \sigma^{3}:-\frac{1}{R} \varepsilon\left(\mu^{2}+r\right)\left(12 m^{2} A_{1} B_{1}^{2} c_{1}-2(1-2 m) \mu \varepsilon B_{1}^{2} a_{1}\right. \\
& -6 m \mu \varepsilon B_{1}^{2} a_{1}+4 m \varepsilon^{2} A_{0} A_{1} a_{1} \\
& \left.+48 m^{2} A_{0} A_{1} B_{1}^{2} c_{1} k_{1}\right)+4 m^{2} A_{1}^{3} c_{1} \\
& +16 m^{2} A_{0} A_{1}^{3} c_{1} k_{1}-2 m \mu \varepsilon A_{1}^{2} a_{1}-\frac{4}{R} \mu \varepsilon^{2}\left(\mu^{2}+r\right) \\
& \cdot\left(4 c_{1} k_{1} m^{2} B_{1}^{4}+4 a_{1} m \varepsilon^{2} B_{1}^{2}+(1-2 m) a_{1} \varepsilon^{2} B_{1}^{2}\right) \\
& +2 \mu \varepsilon\left(24 c_{1} k_{1} m^{2} A_{1}^{2} B_{1}^{2}+4 a_{1} m \varepsilon^{2} A_{1}^{2}\right. \\
& \left.+(1-2 m) a_{1} \varepsilon^{2} A_{1}^{2}\right)=0 \text {, } \\
& \sigma^{3} \tau: \frac{1}{R} \varepsilon\left(\mu^{2}+r\right)\left(16 A_{1} c_{1} k_{1} m^{2} B_{1}^{3}+8 A_{1} a_{1} m \varepsilon^{2} B_{1}\right. \\
& \left.+2(1-2 m) A_{1} a_{1} \varepsilon^{2} B_{1}\right)-16 m^{2} A_{1}^{3} B_{1} c_{1} k_{1}=0, \\
& \sigma^{2}: 4 m^{2} A_{1}^{2} b_{1}+\varepsilon^{2}\left(6 \mu^{2}+2 r\right)\left(4 c_{1} k_{1} m^{2} B_{1}^{4}\right. \\
& \left.+4 a_{1} m \varepsilon^{2} B_{1}^{2}+(1-2 m) a_{1} \varepsilon^{2} B_{1}^{2}\right)+(1-2 m) \\
& \cdot \mu^{2} B_{1}^{2} a_{1}+2 \mu \varepsilon\left(12 m^{2} A_{1} B_{1}^{2} c_{1}-2(1-2 m) \mu \varepsilon B_{1}^{2} a_{1}\right. \\
& -6 m \mu \varepsilon B_{1}^{2} a_{1}+4 m \varepsilon^{2} A_{0} A_{1} a_{1} \\
& \left.+48 m^{2} A_{0} A_{1} B_{1}^{2} c_{1} k_{1}\right)-\frac{1}{R} \varepsilon\left(\mu^{2}+r\right) \\
& \cdot\left(24 c_{1} k_{1} m^{2} A_{0}^{2} B_{1}^{2}+12 c_{1} m^{2} A_{0} B_{1}^{2}+4 b_{1} m^{2} B_{1}^{2}\right. \\
& \left.+4 R \varepsilon a_{1} m B_{1}^{2}+2(1-2 m) R \varepsilon a_{1} B_{1}^{2}\right) \\
& +24 m^{2} A_{0}^{2} A_{1}^{2} c_{1} k_{1}-2 m \mu \varepsilon A_{0} A_{1} a_{1} \\
& -\varepsilon R\left(24 c_{1} k_{1} m^{2} A_{1}^{2} B_{1}^{2}+4 a_{1} m \varepsilon^{2} A_{1}^{2}\right. \\
& \left.+(1-2 m) a_{1} \varepsilon^{2} A_{1}^{2}\right)+12 m^{2} A_{0} A_{1}^{2} c_{1}+2 R m \varepsilon A_{1}^{2} a_{1} \\
& =0 \text {, }
\end{aligned}
$$

$$
\begin{aligned}
\sigma^{2} \tau & : 2 \varepsilon \mu\left(16 A_{1} c_{1} k_{1} m^{2} B_{1}^{3}+8 A_{1} a_{1} m \varepsilon^{2} B_{1}\right. \\
& \left.+2(1-2 m) A_{1} a_{1} \varepsilon^{2} B_{1}\right)+12 m^{2} A_{1}^{2} B_{1} c_{1}-2(1 \\
& -2 m) \mu \varepsilon A_{1} B_{1} a_{1}-\frac{1}{R} \varepsilon\left(\mu^{2}+r\right)\left(4 m^{2} B_{1}^{3} c_{1}\right. \\
& \left.+16 m^{2} A_{0} B_{1}^{3} c_{1} k_{1}+4 m \varepsilon^{2} A_{0} B_{1} a_{1}\right) \\
& +48 m^{2} A_{0} A_{1}^{2} B_{1} c_{1} k_{1}-8 m \mu \varepsilon A_{1} B_{1} a_{1}=0 \\
\sigma & :-\varepsilon R\left(12 m^{2} A_{1} B_{1}^{2} c_{1}-2(1-2 m) \mu \varepsilon B_{1}^{2} a_{1}\right. \\
& -6 m \mu \varepsilon B_{1}^{2} a_{1}+4 m \varepsilon^{2} A_{0} A_{1} a_{1} \\
& \left.+48 m^{2} A_{0} A_{1} B_{1}^{2} c_{1} k_{1}\right)+2 \varepsilon \mu\left(24 c_{1} k_{1} m^{2} A_{0}^{2} B_{1}^{2}\right. \\
& +12 c_{1} m^{2} A_{0} B_{1}^{2}+4 b_{1} m^{2} B_{1}^{2}+4 R \varepsilon a_{1} m B_{1}^{2}
\end{aligned}
$$

$$
\begin{aligned}
& \left.+2(1-2 m) R \varepsilon a_{1} B_{1}^{2}\right)+8 m^{2} A_{0} A_{1} b_{1} \\
& +12 m^{2} A_{0}^{2} A_{1} c_{1}-4 R \mu \varepsilon^{2}\left(4 c_{1} k_{1} m^{2} B_{1}^{4}+4 a_{1} m \varepsilon^{2} B_{1}^{2}\right. \\
& \left.+(1-2 m) a_{1} \varepsilon^{2} B_{1}^{2}\right)+16 m^{2} A_{0}^{3} A_{1} c_{1} k_{1}-2(1 \\
& -2 m) R \mu B_{1}^{2} a_{1}+2 R m \varepsilon A_{0} A_{1} a_{1}=0, \\
& \sigma \tau: 8 m^{2} A_{1} B_{1} b_{1}-\varepsilon R\left(16 A_{1} c_{1} k_{1} m^{2} B_{1}^{3}\right. \\
& \left.+8 \mathrm{~A}_{1} a_{1} m \varepsilon^{2} B_{1}+2(1-2 m) A_{1} a_{1} \varepsilon^{2} B_{1}\right) \\
& +2 \varepsilon \mu\left(4 m^{2} B_{1}^{3} c_{1}+16 m^{2} A_{0} B_{1}^{3} c_{1} k_{1}\right. \\
& \left.+4 m \varepsilon^{2} A_{0} B_{1} a_{1}\right)+24 m^{2} A_{0} A_{1} B_{1} c_{1}+2(1-2 m) \\
& \text { - Re } A_{1} B_{1} a_{1}+6 R m \varepsilon A_{1} B_{1} a_{1}-6 m \mu \varepsilon A_{0} B_{1} a_{1} \\
& +48 m^{2} A_{0}^{2} A_{1} B_{1} c_{1} k_{1}=0 \text {, } \\
& \tau: 8 m^{2} A_{0} B_{1} b_{1}-R \varepsilon\left(4 m^{2} B_{1}^{3} c_{1}+16 m^{2} A_{0} B_{1}^{3} c_{1} k_{1}\right. \\
& \left.+4 m \varepsilon^{2} A_{0} B_{1} a_{1}\right)+12 m^{2} A_{0}^{2} B_{1} c_{1}+16 m^{2} A_{0}^{3} B_{1} c_{1} k_{1} \\
& +4 R m \varepsilon A_{0} B_{1} a_{1}=0 \text {, } \\
& \sigma^{0}: R^{2} \varepsilon^{2}\left(4 c_{1} k_{1} m^{2} B_{1}^{4}+4 a_{1} m \varepsilon^{2} B_{1}^{2}+(1-2 m) a_{1} \varepsilon^{2} B_{1}^{2}\right) \\
& +4 m^{2} A_{0}^{2} b_{1}+4 m^{2} A_{0}^{3} c_{1}+(1-2 m) R^{2} B_{1}^{2} a_{1} \\
& -R \varepsilon\left(24 c_{1} k_{1} m^{2} A_{0}^{2} B_{1}^{2}+12 c_{1} m^{2} A_{0} B_{1}^{2}+4 b_{1} m^{2} B_{1}^{2}\right. \\
& \left.+4 R \varepsilon a_{1} m B_{1}^{2}+2(1-2 m) R \varepsilon a_{1} B_{1}^{2}\right)+4 m^{2} A_{0}^{4} c_{1} k_{1} \\
& =0 \text {. }
\end{aligned}
$$

According to Step 5, of Section 2, there are three cases of solutions of the algebraic equations (26) to be discussed as follows:

Case $1(\varepsilon=-1, r=-1)$. It leads to the following results.

Result 1. We have

$$
\begin{aligned}
A_{0} & =-\frac{b_{1}(m+1)}{c_{1}}, \\
A_{1} & =0, \\
B_{1} & = \pm \frac{(m+1) \sqrt{-b_{1} a_{1}}}{c_{1} m}, \\
\mu & =0, \\
R & =-\frac{b_{1} m^{2}}{a_{1}}, \\
k_{1} & =\frac{c_{1}(2 m+1)}{4 b_{1}(m+1)^{2}} .
\end{aligned}
$$


From (11), (25), and (27), we deduce the kink-shaped soliton solutions of (1) as follows:

$$
\begin{aligned}
& q_{11}(x, t)=\left\{-\frac{b_{1}(m+1)}{c_{1}}(1\right. \\
& \left.\left. \pm \tanh \left(\sqrt{-\frac{b_{1} m^{2}}{a_{1}} \xi}\right)\right)\right\}^{1 / 2 m} \\
& \cdot \exp [i(-k x+\omega t+\theta)]
\end{aligned}
$$

where $a_{1} b_{1}<0$ and $c_{1} b_{1}<0$.

Result 2. We have

$$
\begin{aligned}
A_{0} & =0, \\
A_{1} & =\frac{a_{1} \mu(m+1)}{2 c_{1} m^{2}}, \\
B_{1} & =0, \\
R & =-\frac{4 b_{1} m^{2}}{a_{1}}, \\
k_{1} & =\frac{c_{1}\left(\mu^{2}-1\right)(2 m+1)}{4 b_{1} \mu^{2}(m+1)^{2}} .
\end{aligned}
$$

In this result, we deduce the bell-shaped soliton solutions of (1) as follows:

$$
\begin{aligned}
& q_{12}(x, t) \\
& =\left\{-\frac{2 b_{1} \mu(m+1)}{c_{1}}\left(\frac{\operatorname{sech}\left(\sqrt{-\left(4 b_{1} m^{2} / a_{1}\right)} \xi\right)}{\mu \operatorname{sech}\left(\sqrt{-\left(4 b_{1} m^{2} / a_{1}\right)} \xi\right)+1}\right)\right\}^{1 / 2 m} \\
& \cdot \exp [i(-k x+\omega t+\theta)],
\end{aligned}
$$

where $a_{1} b_{1}<0$ and $c_{1} b_{1} \mu<0$.

Result 3. We have

$$
\begin{aligned}
A_{0} & =-\frac{b_{1}(m+1)}{c_{1}}, \\
A_{1} & = \pm \frac{a_{1}(m+1) \sqrt{\mu^{2}-1}}{4 c_{1} m^{2}} \\
B_{1} & = \pm \frac{(m+1) \sqrt{-a_{1} b_{1}}}{2 c_{1} m} \\
R & =-\frac{4 b_{1} m^{2}}{a_{1}} \\
k_{1} & =\frac{c_{1}(2 m+1)}{4 b_{1}(m+1)^{2}} .
\end{aligned}
$$

In this result, we deduce the bell-kink-shaped soliton solutions of (1) as follows:

$$
\begin{aligned}
& q_{13}(x, t)=\left\{-\frac{b_{1}(m+1)}{c_{1}}(1\right. \\
& \pm \frac{\sqrt{\mu^{2}-1} \operatorname{sech}\left(\sqrt{-\left(4 b_{1} m^{2} / a_{1}\right)} \xi\right)}{\mu \operatorname{sech}\left(\sqrt{-\left(4 b_{1} m^{2} / a_{1}\right)} \xi\right)+1} \\
& \left.\left. \pm \frac{\tanh \left(\sqrt{-\left(4 b_{1} m^{2} / a_{1}\right)} \xi\right)}{\mu \operatorname{sech}\left(\sqrt{-\left(4 b_{1} m^{2} / a_{1}\right)} \xi\right)+1}\right)\right\}^{1 / 2 m} \\
& \cdot \exp [i(-k x+\omega t+\theta)],
\end{aligned}
$$

where $a_{1} b_{1}<0, c_{1} b_{1}<0$ and $\mu^{2}>1$.

Case $2(\varepsilon=-1, r=1)$. It leads to the following results.

Result 1. We have the same result (27).

From (12), (25), and (27), we deduce the singular kinkshaped soliton solutions of (1) as follows:

$$
\begin{aligned}
& q_{21}(x, t) \\
& =\left\{-\frac{b_{1}(m+1)}{c_{1}}\left(1 \pm \operatorname{coth}\left(\sqrt{-\frac{b_{1} m^{2}}{a_{1}} \xi}\right)\right)\right\}^{1 / 2 m} \\
& \quad \cdot \exp [i(-k x+\omega t+\theta)],
\end{aligned}
$$

where $a_{1} b_{1}<0$ and $c_{1} b_{1}<0$.

Result 2. We have

$$
\begin{aligned}
A_{0} & =0, \\
A_{1} & =\frac{a_{1} \mu(m+1)}{2 c_{1} m^{2}}, \\
B_{1} & =0, \\
R & =-\frac{4 b_{1} m^{2}}{a_{1}}, \\
k_{1} & =\frac{c_{1}\left(\mu^{2}+1\right)(2 m+1)}{4 b_{1} \mu^{2}(m+1)^{2}} .
\end{aligned}
$$

In this result, we deduce the singular bell-shaped soliton solutions of (1) as follows:

$$
\begin{aligned}
& q_{22}(x, t) \\
& \quad=\left\{-\frac{2 b_{1} \mu(m+1)}{c_{1}}\left(\frac{\operatorname{csch}\left(\sqrt{-\left(4 b_{1} m^{2} / a_{1}\right)} \xi\right)}{\left.\mu \operatorname{csch}\left(\sqrt{-\left(4 b_{1} m^{2} / a_{1}\right.}\right) \xi\right)+1}\right)\right\}^{1 / 2 m}
\end{aligned}
$$$$
\cdot \exp [i(-k x+\omega t+\theta)] \text {, }
$$

where $a_{1} b_{1}<0$ and $c_{1} b_{1} \mu<0$. 
Result 3. We have

$$
\begin{aligned}
A_{0} & =-\frac{b_{1}(m+1)}{c_{1}}, \\
A_{1} & = \pm \frac{a_{1}(m+1) \sqrt{\mu^{2}+1}}{4 c_{1} m^{2}}, \\
B_{1} & = \pm \frac{(m+1) \sqrt{-a_{1} b_{1}}}{2 c_{1} m}, \\
R & =-\frac{4 b_{1} m^{2}}{a_{1}}, \\
k_{1} & =\frac{c_{1}(2 m+1)}{4 b_{1}(m+1)^{2}} .
\end{aligned}
$$

In this result, we deduce the singular bell-kink-shaped soliton solutions of (1) as follows:

$$
\begin{aligned}
& q_{23}(x, t)=\left\{-\frac{b_{1}(m+1)}{c_{1}}(1\right. \\
& \pm \frac{\sqrt{\mu^{2}+1} \operatorname{csch}\left(\sqrt{-\left(4 b_{1} m^{2} / a_{1}\right)} \xi\right)}{\mu \operatorname{csch}\left(\sqrt{-\left(4 b_{1} m^{2} / a_{1}\right)} \xi\right)+1} \\
& \left.\left. \pm \frac{\operatorname{coth}\left(\sqrt{-\left(4 b_{1} m^{2} / a_{1}\right)} \xi\right)}{\mu \operatorname{csch}\left(\sqrt{-\left(4 b_{1} m^{2} / a_{1}\right)} \xi\right)+1}\right)\right\}^{1 / 2 m} \\
& \cdot \exp [i(-k x+\omega t+\theta)],
\end{aligned}
$$

where $a_{1} b_{1}<0$ and $c_{1} b_{1}<0$.

Case $3(\varepsilon=1, r=-1)$. It leads to the following results.

Result 1. We have

$$
\begin{aligned}
& A_{0}=0, \\
& A_{1}=-\frac{a_{1} \mu(m+1)}{2 c_{1} m^{2}}, \\
& B_{1}=0, \\
& R=\frac{4 b_{1} m^{2}}{a_{1}}, \\
& k_{1}=\frac{c_{1}\left(\mu^{2}-1\right)(2 m+1)}{4 b_{1} \mu^{2}(m+1)^{2}} .
\end{aligned}
$$

From (13), (25), and (38), we deduce the periodic solutions of (1) as follows:

$$
\begin{aligned}
& q_{31}(x, t) \\
& =\left\{-\frac{2 b_{1} \mu(m+1)}{c_{1}}\left(\frac{\sec \left(\sqrt{\left(4 b_{1} m^{2} / a_{1}\right)}\right)}{\mu \sec \left(\sqrt{\left(4 b_{1} m^{2} / a_{1}\right)}\right)+1}\right)\right\}^{1 / 2 m} \\
& \quad \cdot \exp [i(-k x+\omega t+\theta)], \\
& q_{32}(x, t) \\
& \quad=\left\{-\frac{2 b_{1} \mu(m+1)}{c_{1}}\left(\frac{\csc \left(\sqrt{\left(4 b_{1} m^{2} / a_{1}\right)}\right)}{\mu \csc \left(\sqrt{\left(4 b_{1} m^{2} / a_{1}\right)} \xi\right)+1}\right)\right\}^{1 / 2 m} \\
& \quad \cdot \exp [i(-k x+\omega t+\theta)],
\end{aligned}
$$

where $a_{1} b_{1}>0$ and $c_{1} b_{1} \mu<0$.

Remark 1. Note that our results (28) and (33) are in agreement with the results obtained in [5], while the other results are new, which are not found elsewhere.

Remark 2. Note that, if $R=\mu=0$, then we have the trivial solution.

3.2. On Solving (2) Using the Method of Section 2. In this subsection, we apply the generalized projective Riccati equations method of Section 2 to find new soliton and periodic solutions of (2). To this aim, we use the following wave transformation:

$$
U(X, Y, Z, T)=U(\xi), \quad \xi=\alpha X+\beta Y+\gamma Z+v T,
$$

to reduce (2) to the following nonlinear ODE:

$$
V U^{\prime}-A U U^{\prime}-\left(B \gamma^{2}+C\left(\alpha^{2}+\beta^{2}\right)\right) U^{\prime \prime \prime}=0,
$$

where $\alpha, \beta, \gamma$ are the direction cosines, and $v$ is the quantum ion-acoustic wave speed and $V=v / \gamma$. Integrating (42) with respect to $\xi$ once and vanishing the constant of integration, we find the following nonlinear ODE:

$$
V U-\frac{1}{2} A U^{2}-\left(B \gamma^{2}+C\left(\alpha^{2}+\beta^{2}\right)\right) U^{\prime \prime}=0
$$

Balancing $U^{\prime \prime}$ with $U^{2}$ gives $N=2$. Therefore, (6) reduces to

$$
\begin{aligned}
U(\xi)= & A_{0}+A_{1} \sigma(\xi)+A_{2} \sigma^{2}(\xi)+B_{1} \tau(\xi) \\
& +B_{2} \sigma(\xi) \tau(\xi)
\end{aligned}
$$

where $A_{i}(i=0,1,2), B_{1}$ and $B_{2}$ are constants to be determined such that $A_{2} \neq 0$ or $B_{2} \neq 0$.

Substituting (44) and using (7) and (8) into (43), the lefthand side of (43) becomes a polynomial in $\sigma(\xi)$ and $\tau(\xi)$. 
Setting the coefficients of this polynomial to be zero yields the following system of algebraic equations:

$$
\begin{aligned}
& \sigma^{4}: \frac{1}{2} \frac{A}{R} \varepsilon B_{2}^{2}\left(\mu^{2}+r\right)-\frac{1}{2} A A_{2}^{2}=0, \\
& \sigma^{3}: 2\left(B \gamma^{2}+C\left(\alpha^{2}+\beta^{2}\right)\right) \mu \varepsilon A_{2}-A A_{1} A_{2} \\
& -\frac{1}{2} A \varepsilon\left(2 \mu B_{2}^{2}-\frac{2}{R} B_{1} B_{2}\left(\mu^{2}+r\right)\right)=0, \\
& \sigma^{3} \tau: A A_{2} B_{2} \\
& -\frac{4}{R} \varepsilon^{3} B_{2}\left(\mu^{2}+r\right)\left(B \gamma^{2}+C\left(\alpha^{2}+\beta^{2}\right)\right)=0, \\
& \sigma^{2}: V A_{2}-\frac{1}{2} A\left(A_{1}^{2}+2 A_{0} A_{2}\right) \\
& +\frac{1}{2} A \varepsilon\left(R B_{2}^{2}+\frac{1}{R} B_{1}^{2}\left(\mu^{2}+r\right)-4 \mu B_{1} B_{2}\right)=0, \\
& \sigma^{2} \tau: A\left(A_{1} B_{2}+A_{2} B_{1}\right) \\
& +\left(B \gamma^{2}+C\left(\alpha^{2}+\beta^{2}\right)\right)\left(\varepsilon^{2} A_{1}-6 \mu \varepsilon B_{2}\right) \\
& +8\left(B \gamma^{2}+C\left(\alpha^{2}+\beta^{2}\right)\right) \mu \varepsilon^{3} B_{2}=0, \\
& \sigma: V A_{1}-\frac{1}{2} A \varepsilon\left(2 \mu B_{1}^{2}-2 R B_{1} B_{2}\right)-A A_{0} A_{1}=0, \\
& \sigma \tau: V B_{2}-\left(B \gamma^{2}+C\left(\alpha^{2}+\beta^{2}\right)\right)\left(5 R \varepsilon B_{2}-3 \mu \varepsilon B_{1}\right) \\
& -A\left(A_{0} B_{2}+A_{1} B_{1}\right) \\
& +4\left(B \gamma^{2}+C\left(\alpha^{2}+\beta^{2}\right)\right) R \varepsilon^{3} B_{2}=0, \\
& \tau: A A_{0} B_{1}-V B_{1}+2\left(B \gamma^{2}+C\left(\alpha^{2}+\beta^{2}\right)\right) R \varepsilon B_{1}=0, \\
& \sigma^{0}: V A_{0}-\frac{1}{2} A A_{0}^{2}-\left(B \gamma^{2}+C\left(\alpha^{2}+\beta^{2}\right)\right) \varepsilon B_{2} \\
& +\frac{1}{2} A R \varepsilon B_{1}^{2}=0
\end{aligned}
$$

According to Step 5 of Section 2, there are three cases of solutions of the algebraic equations (45) to be discussed as follows.

Case $1(\varepsilon=-1, r=-1)$. It leads to the following results.

Result 1. We have

$$
\begin{aligned}
& A_{0}=0, \\
& A_{1}=\frac{6 \mu\left(B \gamma^{2}+C\left(\alpha^{2}+\beta^{2}\right)\right)}{A}, \\
& A_{2}=-\frac{6\left(\mu^{2}-1\right)\left(B \gamma^{2}+C\left(\alpha^{2}+\beta^{2}\right)\right)}{A R}, \\
& B_{1}=0,
\end{aligned}
$$

$$
\begin{aligned}
& B_{2}= \pm \frac{6 \sqrt{\mu^{2}-1}\left(B \gamma^{2}+C\left(\alpha^{2}+\beta^{2}\right)\right)}{A \sqrt{R}} \\
& V=R\left(B \gamma^{2}+C\left(\alpha^{2}+\beta^{2}\right)\right) .
\end{aligned}
$$

From (11), (44), and (46), we deduce the bell-kink-shaped soliton solution of (2) as follows:

$$
\begin{aligned}
& U_{11}(X, Y, Z, T)=\left\{\frac{6\left(B \gamma^{2}+C\left(\alpha^{2}+\beta^{2}\right)\right)}{A}\right. \\
& . \frac{R \operatorname{sech}(\sqrt{R} \xi)}{\mu \operatorname{sech}(\sqrt{R} \xi)+1}\left(\frac{\left(1-\mu^{2}\right) \operatorname{sech}(\sqrt{R} \xi)}{\mu \operatorname{sech}(\sqrt{R} \xi)+1}\right. \\
& \left.\left. \pm \frac{\sqrt{\mu^{2}-1} \tanh (\sqrt{R} \xi)}{\mu \operatorname{sech}(\sqrt{R} \xi)+1}+\mu\right)\right\},
\end{aligned}
$$

where $R>0, \mu^{2}>1$ and $\xi=\alpha X+\beta Y+\gamma Z+\left[R \gamma\left(B \gamma^{2}+\right.\right.$ $\left.\left.C\left(\alpha^{2}+\beta^{2}\right)\right)\right] T$.

Result 2. We have

$$
\begin{aligned}
& A_{0}=-\frac{2 R\left(B \gamma^{2}+C\left(\alpha^{2}+\beta^{2}\right)\right)}{A}, \\
& A_{1}=\frac{6 \mu\left(B \gamma^{2}+C\left(\alpha^{2}+\beta^{2}\right)\right)}{A}, \\
& A_{2}=-\frac{6\left(\mu^{2}-1\right)\left(B \gamma^{2}+C\left(\alpha^{2}+\beta^{2}\right)\right)}{A R}, \\
& B_{1}=0, \\
& B_{2}= \pm \frac{6 \sqrt{\mu^{2}-1}\left(B \gamma^{2}+C\left(\alpha^{2}+\beta^{2}\right)\right)}{A R}, \\
& V=-R\left(B \gamma^{2}+C\left(\alpha^{2}+\beta^{2}\right)\right) .
\end{aligned}
$$

In this result, we deduce the bell-kink-shaped soliton solution of (2) as follows:

$$
U_{12}(X, Y, Z, T)=\left\{\frac{6\left(B \gamma^{2}+C\left(\alpha^{2}+\beta^{2}\right)\right)}{A}\right.
$$

$$
\begin{aligned}
& \cdot \frac{R \operatorname{sech}(\sqrt{R} \xi)}{\mu \operatorname{sech}(\sqrt{R} \xi)+1}\left(\frac{\left(1-\mu^{2}\right) \operatorname{sech}(\sqrt{R} \xi)}{\mu \operatorname{sech}(\sqrt{R} \xi)+1}\right. \\
& \left. \pm \frac{\sqrt{\mu^{2}-1} \tanh (\sqrt{R} \xi)}{\mu \operatorname{sech}(\sqrt{R} \xi)+1}+\mu\right) \\
& \left.-\frac{2 R\left(B \gamma^{2}+C\left(\alpha^{2}+\beta^{2}\right)\right)}{A}\right\},
\end{aligned}
$$


where $R>0, \mu^{2}>1$ and $\xi=\alpha X+\beta Y+\gamma Z-\left[R \gamma\left(B \gamma^{2}+\right.\right.$ $\left.\left.C\left(\alpha^{2}+\beta^{2}\right)\right)\right] T$.

Case $2(\varepsilon=-1, r=1)$. It leads to the following results:

Result 1. We have

$$
\begin{aligned}
& A_{0}=0, \\
& A_{1}=\frac{6 \mu\left(B \gamma^{2}+C\left(\alpha^{2}+\beta^{2}\right)\right)}{A}, \\
& A_{2}=-\frac{6\left(\mu^{2}+1\right)\left(B \gamma^{2}+C\left(\alpha^{2}+\beta^{2}\right)\right)}{A R}, \\
& B_{1}=0, \\
& B_{2}= \pm \frac{6 \sqrt{\mu^{2}+1}\left(B \gamma^{2}+C\left(\alpha^{2}+\beta^{2}\right)\right)}{A \sqrt{R}}, \\
& V=R\left(B \gamma^{2}+C\left(\alpha^{2}+\beta^{2}\right)\right) .
\end{aligned}
$$

From (12), (44), and (50), we deduce the singular bell-kinkshaped soliton solution of (2) as follows:

$$
\begin{aligned}
& U_{21}(X, Y, Z, T)=\left\{\frac{6\left(B \gamma^{2}+C\left(\alpha^{2}+\beta^{2}\right)\right)}{A}\right. \\
& \cdot \frac{R \operatorname{csch}(\sqrt{R} \xi)}{\mu \operatorname{csch}(\sqrt{R} \xi)+1}\left(-\frac{\left(\mu^{2}+1\right) \operatorname{csch}(\sqrt{R} \xi)}{\mu \operatorname{csch}(\sqrt{R} \xi)+1}\right. \\
& \left.\left. \pm \frac{\sqrt{\mu^{2}+1} \operatorname{coth}(\sqrt{R} \xi)}{\mu \operatorname{csch}(\sqrt{R} \xi)+1}+\mu\right)\right\},
\end{aligned}
$$

where $R>0$ and $\xi=\alpha X+\beta Y+\gamma Z+\left[R \gamma\left(B \gamma^{2}+C\left(\alpha^{2}+\beta^{2}\right)\right)\right] T$. Result 2. We have

$$
\begin{aligned}
& A_{0}=-\frac{2 R\left(B \gamma^{2}+C\left(\alpha^{2}+\beta^{2}\right)\right)}{A}, \\
& A_{1}=\frac{6 \mu\left(B \gamma^{2}+C\left(\alpha^{2}+\beta^{2}\right)\right)}{A}, \\
& A_{2}=-\frac{6\left(\mu^{2}+1\right)\left(B \gamma^{2}+C\left(\alpha^{2}+\beta^{2}\right)\right)}{A R}, \\
& B_{1}=0, \\
& B_{2}= \pm \frac{6 \sqrt{\mu^{2}+1}\left(B \gamma^{2}+C\left(\alpha^{2}+\beta^{2}\right)\right)}{A R}, \\
& V=-R\left(B \gamma^{2}+C\left(\alpha^{2}+\beta^{2}\right)\right) .
\end{aligned}
$$

In this result, we deduce the singular bell-kink-shaped soliton solution of (2) as follows:

$$
\begin{aligned}
& U_{22}(X, Y, Z, T)=\left\{\frac{6\left(B \gamma^{2}+C\left(\alpha^{2}+\beta^{2}\right)\right)}{A}\right. \\
& \cdot \frac{R \operatorname{csch}(\sqrt{R} \xi)}{\mu \operatorname{csch}(\sqrt{R} \xi)+1}\left(-\frac{\left(\mu^{2}+1\right) \operatorname{csch}(\sqrt{R} \xi)}{\mu \operatorname{csch}(\sqrt{R} \xi)+1}\right. \\
& \left. \pm \frac{\sqrt{\mu^{2}+1} \operatorname{coth}(\sqrt{R} \xi)}{\mu \operatorname{csch}(\sqrt{R} \xi)+1}+\mu\right) \\
& \left.-\frac{2 R\left(B \gamma^{2}+C\left(\alpha^{2}+\beta^{2}\right)\right)}{A}\right\},
\end{aligned}
$$

where $R>0$ and $\xi=\alpha X+\beta Y+\gamma Z-\left[R \gamma\left(B \gamma^{2}+C\left(\alpha^{2}+\beta^{2}\right)\right)\right] T$. Case $3(\varepsilon=1, r=-1)$. It leads to the following results.

Result 1. We have

$$
\begin{aligned}
& A_{0}=0, \\
& A_{1}=-\frac{6 \mu\left(B \gamma^{2}+C\left(\alpha^{2}+\beta^{2}\right)\right)}{A}, \\
& A_{2}=\frac{6\left(\mu^{2}-1\right)\left(B \gamma^{2}+C\left(\alpha^{2}+\beta^{2}\right)\right)}{A R}, \\
& B_{1}=0, \\
& B_{2}= \pm \frac{6 \sqrt{1-\mu^{2}}\left(B \gamma^{2}+C\left(\alpha^{2}+\beta^{2}\right)\right)}{A \sqrt{R}}, \\
& V=-R\left(B \gamma^{2}+C\left(\alpha^{2}+\beta^{2}\right)\right) .
\end{aligned}
$$

From (13), (44), and (54), we deduce the periodic solutions of (2) as follows:

$$
\begin{aligned}
& U_{31}(X, Y, Z, T)=\left\{\frac{6\left(B \gamma^{2}+C\left(\alpha^{2}+\beta^{2}\right)\right)}{A}\right. \\
& \cdot \frac{R \sec (\sqrt{R} \xi)}{\mu \sec (\sqrt{R} \xi)+1}\left(\frac{\left(\mu^{2}-1\right) \sec (\sqrt{R} \xi)}{\mu \sec (\sqrt{R} \xi)+1}\right. \\
& \left.\left. \pm \frac{\sqrt{1-\mu^{2}} \tan (\sqrt{R} \xi)}{\mu \sec (\sqrt{R} \xi)+1}-\mu\right)\right\}, \\
& U_{32}(X, Y, Z, T)=\left\{\frac{6\left(B \gamma^{2}+C\left(\alpha^{2}+\beta^{2}\right)\right)}{A}\right.
\end{aligned}
$$



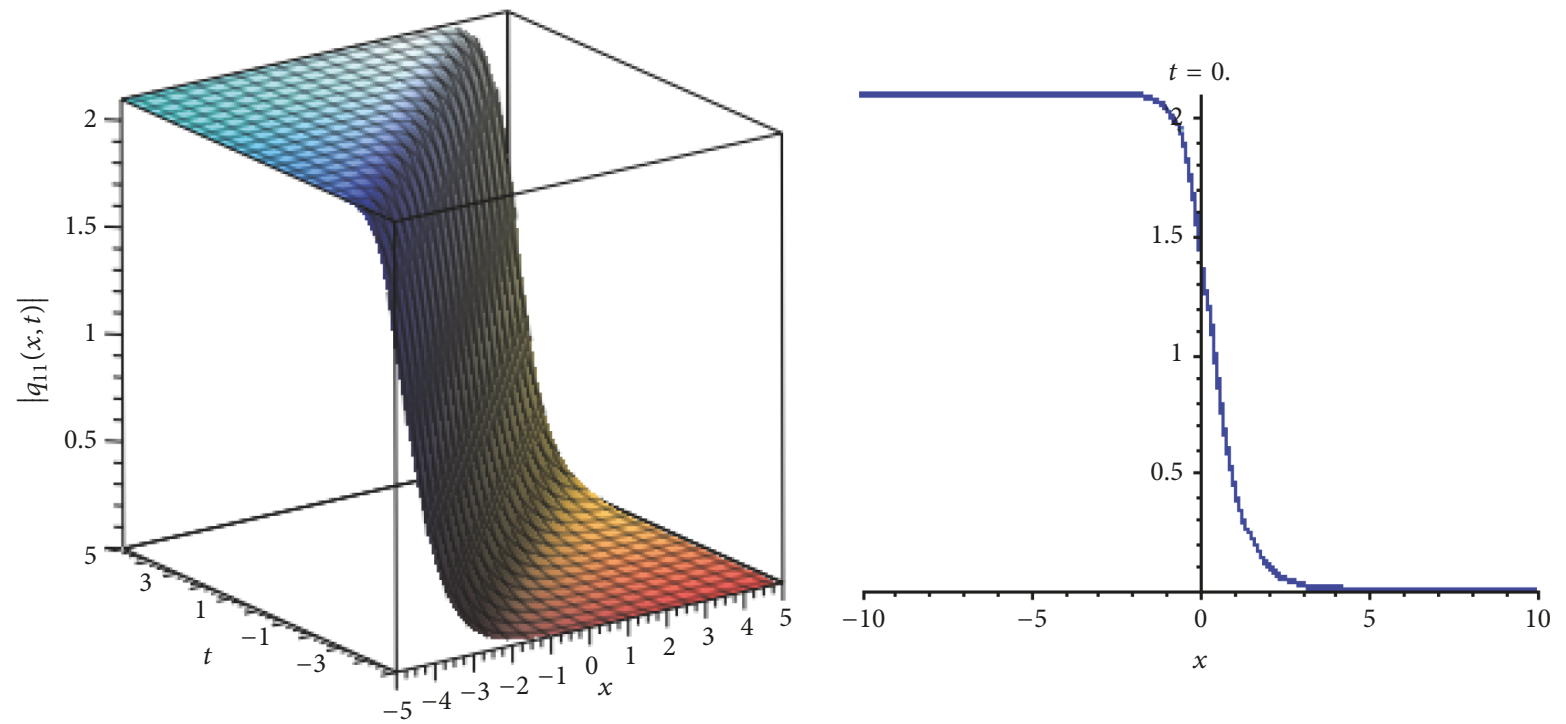

FIgURE 1: Plot solution $\left|q_{11}(x, t)\right|$ of (28) with $a_{1}=-1, b_{1}=11 / 5, c_{1}=-2, m=v=1$.

$$
\begin{aligned}
& \cdot \frac{R \csc (\sqrt{R} \xi)}{\mu \csc (\sqrt{R} \xi)+1}\left(\frac{\left(\mu^{2}-1\right) \csc (\sqrt{R} \xi)}{\mu \csc (\sqrt{R} \xi)+1}\right. \\
& \left.\left. \pm \frac{\sqrt{1-\mu^{2}} \cot (\sqrt{R} \xi)}{\mu \csc (\sqrt{R} \xi)+1}-\mu\right)\right\},
\end{aligned}
$$

where $R>0, \mu^{2}<1$ and $\xi=\alpha X+\beta Y+\gamma Z-\left[R \gamma\left(B \gamma^{2}+\right.\right.$ $\left.\left.C\left(\alpha^{2}+\beta^{2}\right)\right)\right] T$.

Result 2. We have

$$
\begin{aligned}
& A_{0}=\frac{2 R\left(B \gamma^{2}+C\left(\alpha^{2}+\beta^{2}\right)\right)}{A}, \\
& A_{1}=-\frac{6 \mu\left(B \gamma^{2}+C\left(\alpha^{2}+\beta^{2}\right)\right)}{A}, \\
& A_{2}=\frac{6\left(\mu^{2}-1\right)\left(B \gamma^{2}+C\left(\alpha^{2}+\beta^{2}\right)\right)}{A R}, \\
& B_{1}=0, \\
& B_{2}= \pm \frac{6 \sqrt{1-\mu^{2}}\left(B \gamma^{2}+C\left(\alpha^{2}+\beta^{2}\right)\right)}{A \sqrt{R}} \\
& V=R\left(B \gamma^{2}+C\left(\alpha^{2}+\beta^{2}\right)\right) .
\end{aligned}
$$

In this result, we deduce the periodic solutions of (2) as follows:

$$
\begin{array}{r}
U_{33}(X, Y, Z, T)=\left\{\frac{6\left(B \gamma^{2}+C\left(\alpha^{2}+\beta^{2}\right)\right)}{A}\right. \\
\cdot \frac{R \sec (\sqrt{R} \xi)}{\mu \sec (\sqrt{R} \xi)+1}\left(\frac{\left(\mu^{2}-1\right) \sec (\sqrt{R} \xi)}{\mu \sec (\sqrt{R} \xi)+1}\right.
\end{array}
$$

$$
\begin{gathered}
\left. \pm \frac{\sqrt{1-\mu^{2}} \tan (\sqrt{R} \xi)}{\mu \sec (\sqrt{R} \xi)+1}-\mu\right) \\
\left.+\frac{2 R\left(B \gamma^{2}+C\left(\alpha^{2}+\beta^{2}\right)\right)}{A}\right\}, \\
U_{34}(X, Y, Z, T)=\left\{\frac{6\left(B \gamma^{2}+C\left(\alpha^{2}+\beta^{2}\right)\right)}{A}\right. \\
\cdot \frac{R \csc (\sqrt{R} \xi)}{\mu \csc (\sqrt{R} \xi)+1}\left(\frac{\left(\mu^{2}-1\right) \csc (\sqrt{R} \xi)}{\mu \csc (\sqrt{R} \xi)+1}\right. \\
\left. \pm \frac{\sqrt{1-\mu^{2}} \cot (\sqrt{R} \xi)}{\mu \csc (\sqrt{R} \xi)+1}-\mu\right) \\
\left.+\frac{2 R\left(B \gamma^{2}+C\left(\alpha^{2}+\beta^{2}\right)\right)}{A}\right\},
\end{gathered}
$$

where $R>0, \mu^{2}<1$ and $\xi=\alpha X+\beta Y+\gamma Z+\left[R \gamma\left(B \gamma^{2}+\right.\right.$ $\left.\left.C\left(\alpha^{2}+\beta^{2}\right)\right)\right] T$.

\section{Graphical Representations of Some Solutions}

In this section, we draw graphs of some exact solutions. The obtained soliton and periodic solutions are kink and antikink solitons, bell (bright) and antibell (dark) solitary wave solutions, and trigonometric solutions.

Let us now examine Figures 1-3 as they illustrate some of our solutions obtained in this article. To this aim, we select 

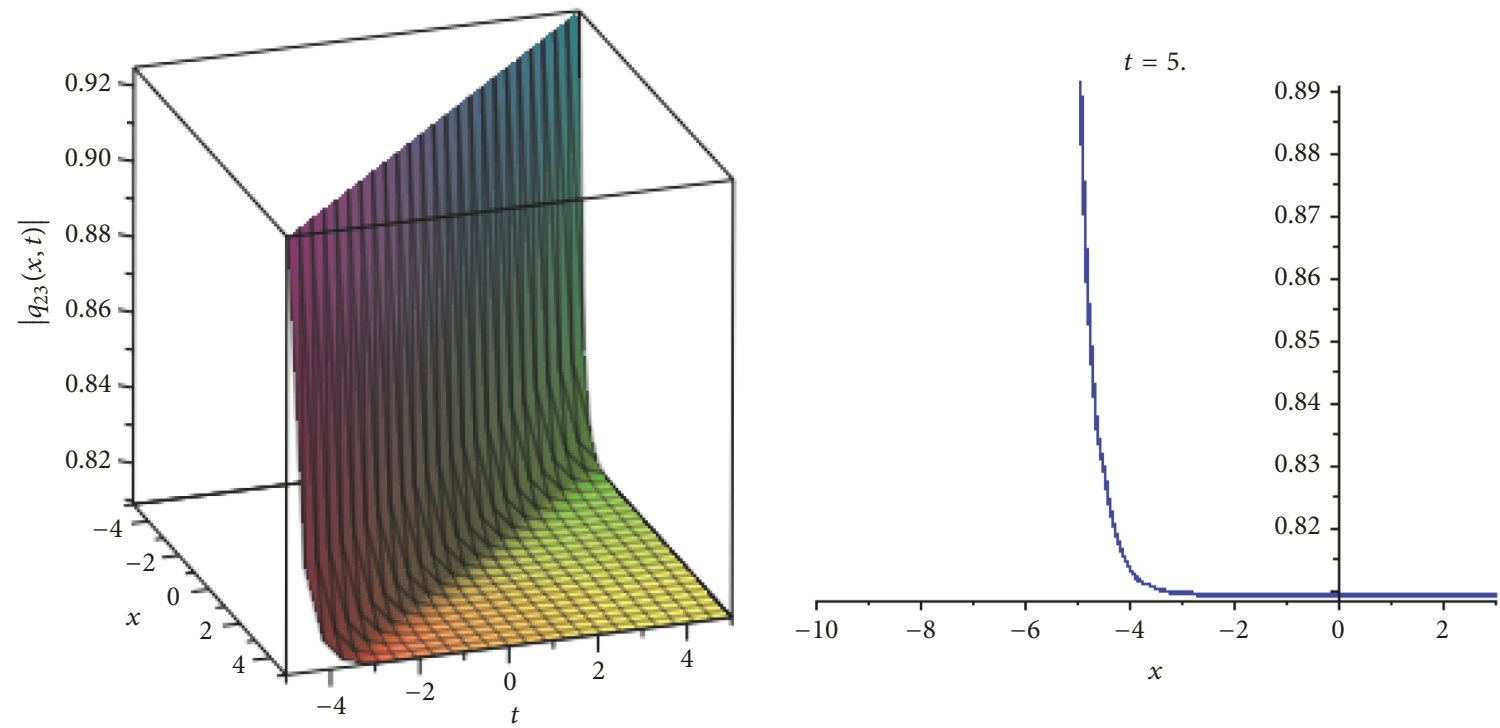

Figure 2: Plot solution $\left|q_{23}(x, t)\right|$ of (37) with $a_{1}=23, b_{1}=-12, c_{1}=168, \mu=1, v=-1, m=2$.
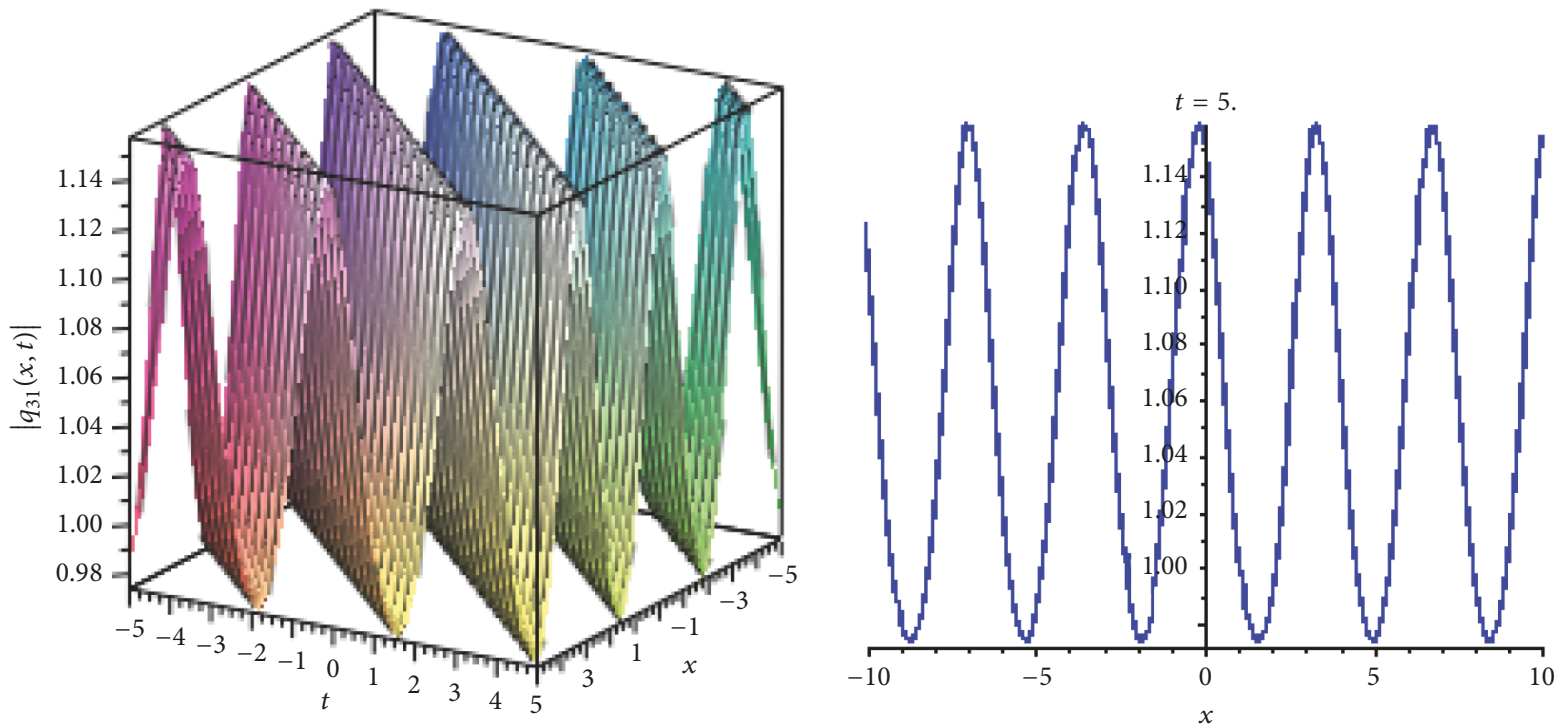

FIGURE 3: Plot solution $\left|q_{31}(x, t)\right|$ of (39) with $a_{1}=-19, b_{1}=-4, c_{1}=20, \mu=3, v=1, m=2$.

some special values of the parameters obtained, for example, in some of the solutions (28), (37), and (39) of the NLS equation with fourth-order dispersion and dual power law nonlinearity (1). For more convenience the graphical representations of these solutions are shown in Figures 1, 2, and 3.

From Figures 1, 2, and 3, one can see that the obtained solutions possess the solitary wave solutions and periodic wave solution. Also, these figures express the behavior of these solutions which give some perspective to readers on how the behavior solutions are produced.

\section{Conclusions}

In this article, based on the generalized projective Riccati equations method described in Section 2, we have obtained many new types of soliton and periodic solutions for the two higher-order nonlinear PDEs, namely, the NLS equation (1) and the nonlinear QZK equation (2). The exact solutions of these equations include kink and antikink solitons, bell (bright) and antibell (dark) solitary wave solutions, and periodic solutions. Comparing our results in this article with the well-known results of $[5,6,21-23]$, we conclude that all our results are new and not found elsewhere except for results (28) and (33) which are in agreement with results (19) and (20) obtained in [5]. The proposed method of this article is effective and can be applied to many other nonlinear PDEs. Finally, all solutions obtained in this article have been checked with the Maple 2015 by putting them back into the original equations (1) and (2). 


\section{Conflicts of Interest}

The authors declare that there are no conflicts of interest regarding the publication of this paper.

\section{References}

[1] M. Wang, X. Li, and J. Zhang, “The $\left(G^{\prime} / G\right)$-expansion method and travelling wave solutions of nonlinear evolution equations in mathematical physics," Physics Letters A, vol. 372, no. 4, pp. 417-423, 2008.

[2] E. M. Zayed, “The $\left(G^{\prime} / G\right)$-expansion method and its applications to some nonlinear evolution equations in the mathematical physics," Applied Mathematics and Computation, vol. 30, no. 1-2, pp. 89-103, 2009.

[3] A. Bekir, "Application of the $\left(G^{\prime} / G\right)$-expansion method for nonlinear evolution equations," Physics Letters A, vol. 372, no. 19, pp. 3400-3406, 2008.

[4] N. A. Kudryashov, "A note on the $\left(G^{\prime} / G\right)$-expansion method," Applied Mathematics and Computation, vol. 217, no. 4, pp. 1755$1758,2010$.

[5] E. M. E. Zayed, A.-G. Al-Nowehy, and M. E. M. Elshater, "Solitons and other solutions to nonlinear Schrödinger equation with fourth-order dispersion and dual power law nonlinearity using several different techniques," The European Physical Journal Plus, vol. 132, no. 6, article no. 259, 2017.

[6] E. M. Zayed and K. A. Alurrfi, "Extended generalized $\left(G^{\prime}\right.$ / $G)$-expansion method for solving the nonlinear quantum Zakharov-Kuznetsov equation," Ricerche di Matematica, vol. 65, no. 1, pp. 235-254, 2016.

[7] G.-q. Xu, "Extended auxiliary equation method and its applications to three generalized NLS equations," Abstract and Applied Analysis, vol. 2014, Article ID 541370, 7 pages, 2014.

[8] E. M. Zayed and K. A. Alurrfi, "Extended auxiliary equation method and its applications for finding the exact solutions for a class of nonlinear Schrödinger-type equations," Applied Mathematics and Computation, vol. 289, pp. 111-131, 2016.

[9] X. Zeng and X. Yong, "A new mapping method and its applications to nonlinear partial differential equations," Physics Letters A, vol. 372, no. 44, pp. 6602-6607, 2008.

[10] E. M. E. Zayed and A.-G. Al-Nowehy, "Solitons and other exact solutions for a class of nonlinear Schrödinger-type equations," Optik - International Journal for Light and Electron Optics, vol. 130, pp. 1295-1311, 2017.

[11] E. M. E. Zayed and K. A. E. Alurrfi, "Solitons and other solutions for two nonlinear Schrödinger equations using the new mapping method," Optik - International Journal for Light and Electron Optics, vol. 144, pp. 132-148, 2017.

[12] R. Conte and M. Musette, "Link between solitary waves and projective Riccati equations," Journal of Physics A: Mathematical and General, vol. 25, no. 21, pp. 5609-5623, 1992.

[13] E. M. Zayed and K. A. Alurrfi, "The generalized projective Riccati equations method for solving nonlinear evolution equations in mathematical physics," Abstract and Applied Analysis, Article ID 259190, Art. ID 259190, 10 pages, 2014.

[14] E. M. E. Zayed and K. A. E. Alurrfi, "The generalized projective Riccati equations method and its applications for solving two nonlinear PDEs describing microtubules," Int. J. Phys. Sci, vol. 10, pp. 391-402, 2015.
[15] G. X. Zhang, Z. B. Li, and Y. S. Duan, "Exact solitary wave solutions of nonlinear wave equations," Science China Mathematics, vol. 44, no. 3, pp. 396-401, 2001.

[16] Z. Y. Yan, "Generalized method and its application in the higher-order nonlinear Schrodinger equation in nonlinear optical fibres," Chaos, Solitons \& Fractals, vol. 16, no. 5, pp. 759766, 2003.

[17] E. Yomba, "The general projective Riccati equations method and exact solutions for a class of nonlinear partial differential equations," Chinese Journal of Physics, vol. 43, no. 6, pp. 9911003, 2005.

[18] E. M. E. Zayed, A. M. Shahoot, and K. A. E. Alurrfi, “The $\left(G^{\prime} / G\right.$, $1 / G)$-expansion method and its applications for constructing many new exact solutions of the higher-order nonlinear Schrö dinger equation and the quantum ZakharovKuznetsov equation," Opt. Quant. Electron, vol. 50, pp. 10-1007, 2018.

[19] T. C. Bountis, V. Papageorgiou, and P. Winternitz, "On the integrability of systems of nonlinear ordinary differential equations with superposition principles," Journal of Mathematical Physics, vol. 27, no. 5, pp. 1215-1224, 1986.

[20] G.-Q. Xu, "New types of exact solutions for the fourth-order dispersive cubic-quintic nonlinear Schrödinger equation," Applied Mathematics and Computation, vol. 217, no. 12, pp. 5967-5971, 2011.

[21] W. M. Moslem, S. Ali, P. K. Shukla, X. Y. Tang, and G. Rowlands, "Solitary, explosive, and periodic solutions of the quantum Zakharov-Kuznetsov equation and its transverse instability," Physics of Plasmas, vol. 14, no. 8, Article ID 082308, 2007.

[22] H. Washimi and T. Taniuti, "Propagation of ion-acoustic solitary waves of small amplitude," Physical Review Letters, vol. 17, no. 19, pp. 996-998, 1966.

[23] B.-g. Zhang, Z.-r. Liu, and Q. Xiao, "New exact solitary wave and multiple soliton solutions of quantum Zakharov-Kuznetsov equation," Applied Mathematics and Computation, vol. 217, no. 1, pp. 392-402, 2010. 


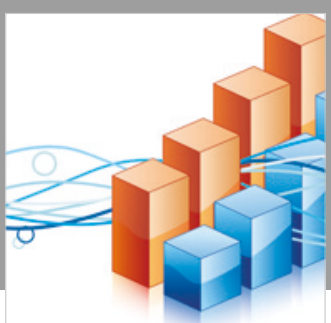

Advances in

Operations Research

\section{-n-m}
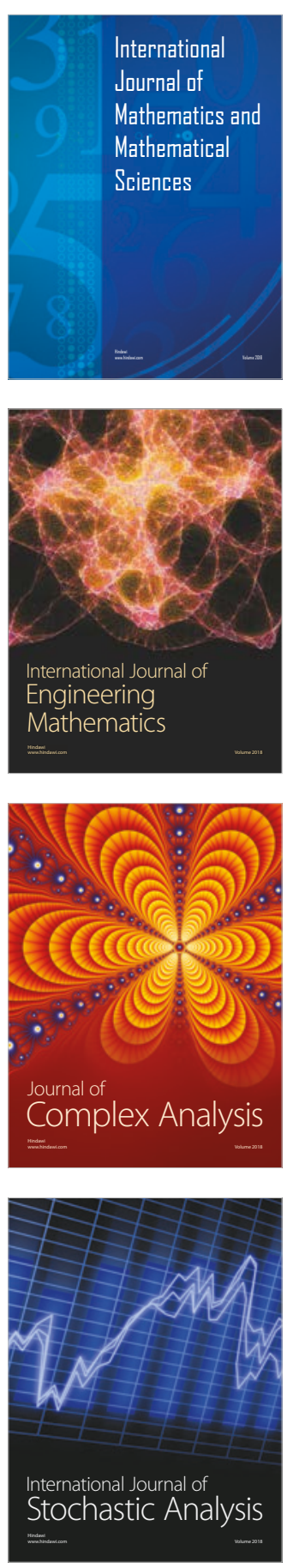
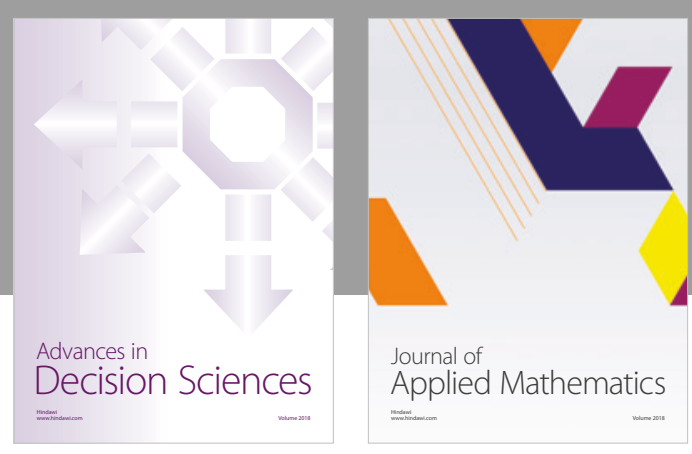

Journal of

Applied Mathematics
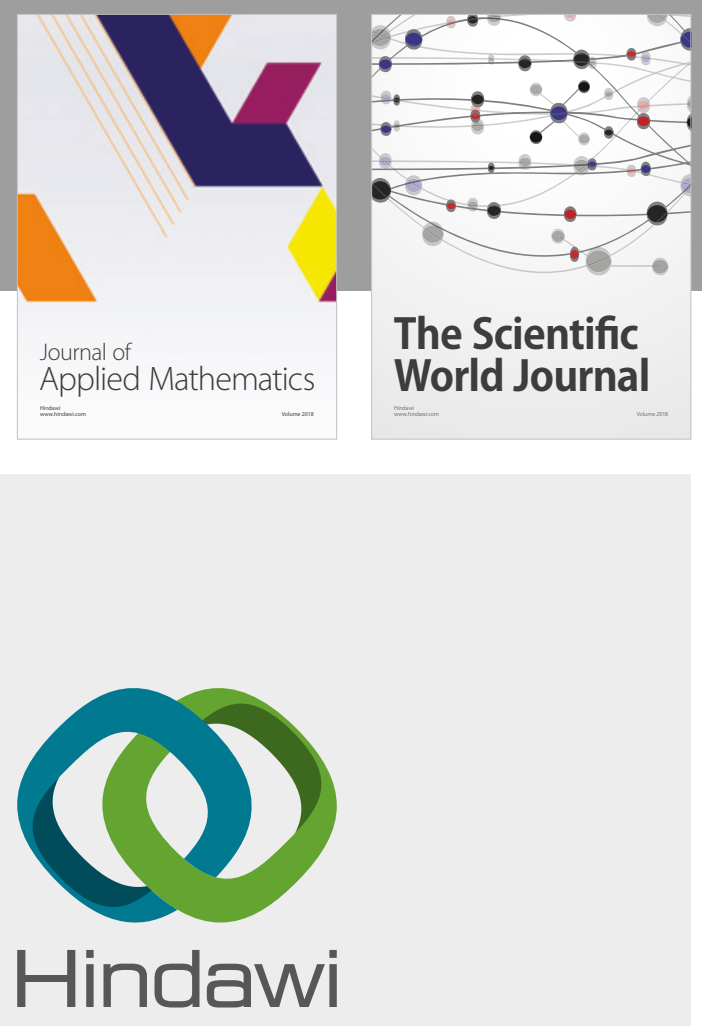

Submit your manuscripts at

www.hindawi.com

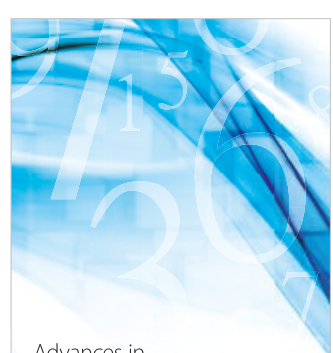

Advances in
Numerical Analysis
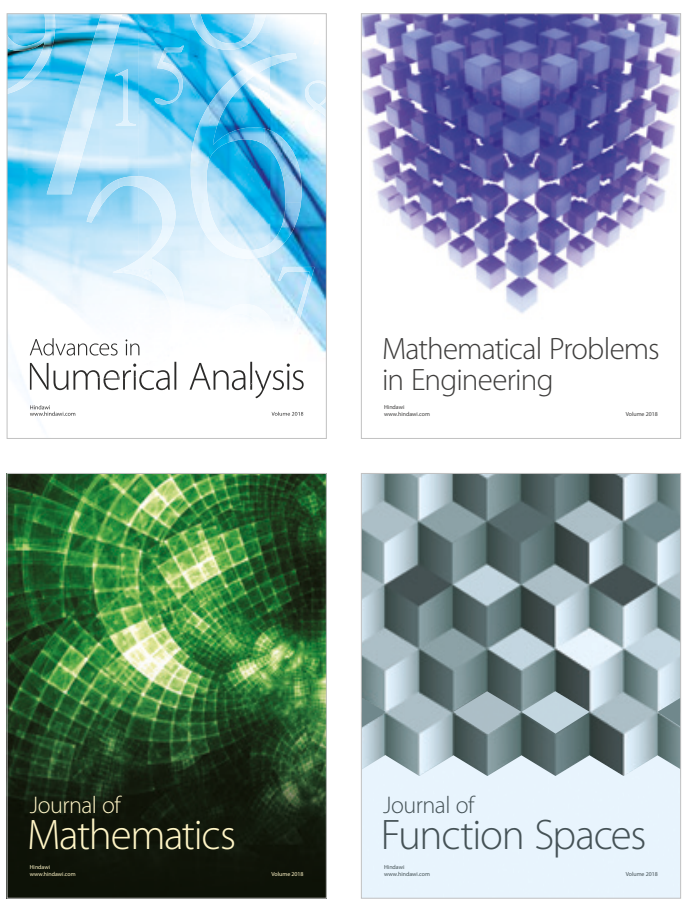

Mathematical Problems in Engineering

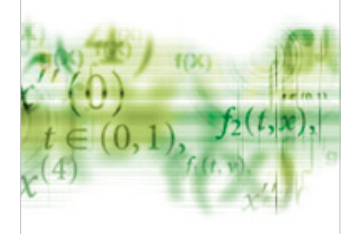

International Journal of

Differential Equations

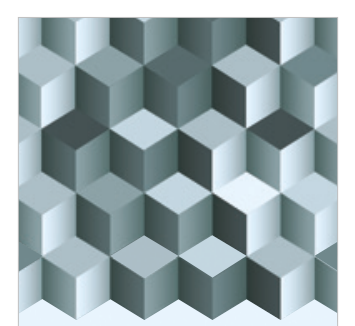

Journal of

Function Spaces

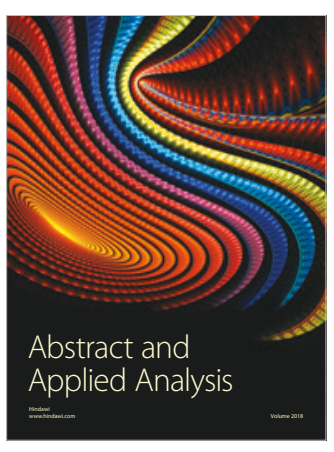

The Scientific

World Journal

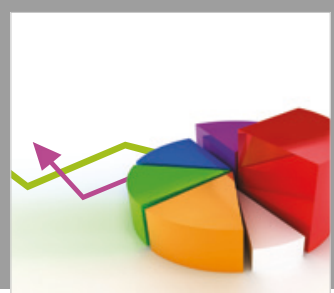

Journal of

Probability and Statistics
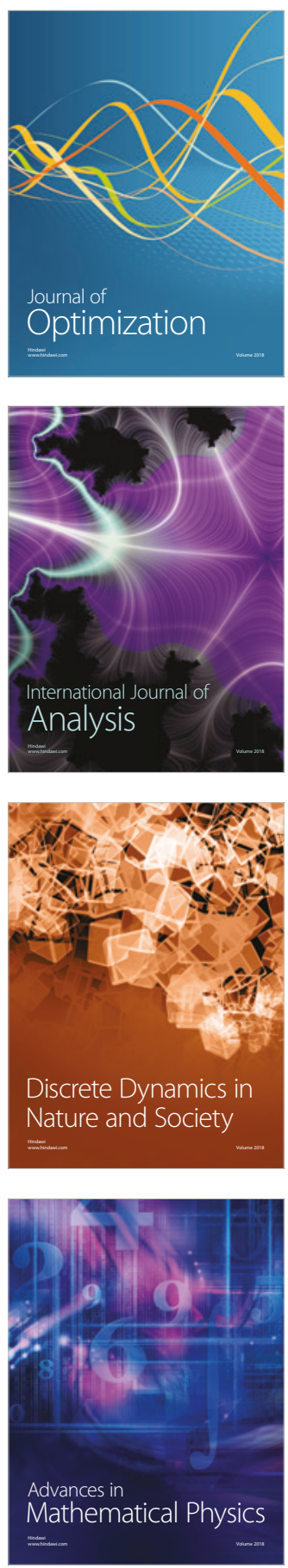